\title{
Efeito da fadiga induzida pelo treino de força na resposta da pressão arterial em sujeitos hipertensos: Uma revisão sistemática
}

\author{
Effect of fatigue induced by strength training on blood pressure response \\ in hypertensive subjects: A systematic review
}

\author{
A.J. Rodrigues-da-Silva, A.A. Lima, S.C. Rodrigues, C.C. Júnior, R.M. Ritti-Dias
}

ARTIGO ORIGINAL | ORIGINAL ARTICLE

\begin{abstract}
O objetivo do estudo foi proceder a uma revisão sistemática da literatura sobre a resposta da pressão arterial após o treino de força em hipertensos, procurando identificar o efeito da fadiga nessa resposta. Foi realizada uma pesquisa bibliográfica nas bases de dados SciELO, LILACS e PubMed/MedLine. Foram encontrados 9377 estudos e apenas seis atenderam aos critérios de inclusão estabelecidos e foram incluídos na revisão. Cinco estudos $(83,3 \%)$ verificaram uma redução na pressão arterial sistólica ou diastólica. Relativamente à fadiga, $66,7 \%(n=4)$ dos estudos relataram que realizaram séries até a fadiga, ao passo que 33,3\% $(n=2)$ relataram que as séries eram interrompidas antes da fadiga. Dos quatro estudos que utilizaram protocolos até a fadiga, 75\% $(n=3)$ observaram redução da pressão arterial, e os dois estudos que interromperam as séries antes da fadiga também evidenciaram redução. A magnitude da redução da pressão arterial foi similar entre os estudos que utilizaram os dois protocolos. Sendo assim, os resultados indicaram que a redução da pressão arterial após o treino de força em sujeitos hipertensos é independente da ocorrência de fadiga.

Palavras-chave: pressão arterial, hipertensão, treino de força, fadiga, exercício
\end{abstract}

ABSTRACT

The objective of this study was to systematically review the literature on blood pressure response after strength training in hypertensive subjects, trying to identify the effect of failure in this response. We performed a literature search in SciELO, LILACS, PubMed / MedLine databases. Out of 9377 studies, only six met the inclusion criteria and were included in the review. Five studies (83.3\%) reported a reduction in systolic or diastolic blood pressure. Regarding failure, $66.7 \%(n=4)$ of the studies performed the sets until failure while $33.3 \%(n=2)$ interrupted exercise before failure. Out of the four studies that used protocols until failure, $75 \%(n=3)$ observed a reduction in blood pressure, and the two studies who discontinued before the series also showed a reduction of failure. The magnitude of blood pressure reduction was similar among studies that employed both protocols. Thus, the results indicated that the reduction in blood pressure after strength training in subjects with hypertension is independent of the occurrence of failure.

Keywords: blood pressure, hypertension, strength training, fatigue, exercise

Submetido: 08.02.2012 | Aceite: 07.12.2012

Anderson José Melo Rodrigues-da-Silva, Aluísio Henrique Rodrigues de Andrade Lima, Sérgio Luiz Cahú Rodrigues, Raphael Mendes Ritti-Dias. Universidade de Pernambuco, Escola Superior de Educação Física, Recife, PE, Brasil.

Crivaldo Gomes Cardoso Júnior. Universidade Estadual de Londrina, Londrina, PR, Brasil.

Endereço para correspondência: Prof. Dr. Raphael Mendes Ritti Dias, Escola Superior de Educação Física, Universidade de Pernambuco, Rua Arnóbio Marques, 310. Santo Amaro, Recife, PE, CEP: 50100-130, Brasil.

E-mail: raphaelritti@gmail.com 
Após a realização do exercício de força ocorre uma série de ajustes cardiovasculares que podem resultar na redução da pressão arterial (PA) para níveis inferiores aos observados no período pré-exercício (Hardy \& Tucker, 1998).

Diversos estudos têm demonstrado que as características dos estímulos físico/motores durante a realização do exercício de força podem influenciar a redução da PA. Deste modo, tem sido demonstrado que essa redução pode apresentar maior magnitude ou perdurar por mais tempo em função da maior massa muscular envolvida (Lizardo \& Simões, 2005; M. D. Polito \& Farinatti, 2009), bem como em função do maior número de séries realizadas (Mediano, Paravidino, Simão, Pontes, \& Polito, 2005). Por outro lado, variáveis como a intensidade utilizada (Lizardo \& Simões, 2005; Rezk, Marrache, Tinucci, Mion, \& Forjaz, 2006) e o intervalo de recuperação (Maior et al., 2007; Veloso et al., 2010) não parecem afetar a diminuição da PA. Embora estes resultados forneçam indicadores importantes para a prescrição do exercício de força direcionado a potencializar os benefícios cardiovasculares em hipertensos, o efeito de outras variáveis importantes que compõem a sessão de treino, como por exemplo, a fadiga muscular, ainda permanece pouco esclarecida.

Evidências têm demonstrado que a realização do exercício de força até à fadiga muscular concêntrica induz um incremento acentuado da PA em hipertensos (de Souza Nery et al., 2010; Gomides, Nery, Júnior, Tinucci, \& Forjaz, 2007). Neste sentido, a VI Diretriz Brasileira de Hipertensão Arterial (2010) recomenda que hipertensos interrompam o exercício de força antes da ocorrência de fadiga. Contudo, é importante realçar, que até ao momento não há relato de desfechos cardiovasculares em conseqüência da realização do exercício de força até à fadiga muscular concêntrica. Por outro lado, é possível que a realização do exercício de força até a fadiga potencie a queda da PA.
Considerando que a redução da PA após o exercício de força tem sido atribuída à redução do débito cardíaco que não é compensada pelo aumento da resistência vascular periférica (Rezk et al., 2006), é possível que a realização do exercício até a fadiga promova uma maior diminuição da PA, uma vez que o exercício até a fadiga induz maior acumulação de metabólitos locais (Willardson, 2007), e isto poderia potencializar a vasodilatação mantida pós-exercício. Entretanto, até a actualidade pouco se sabe sobre o efeito da fadiga nas respostas da PA após o treino de força em hipertensos.

Assim, o objetivo do presente estudo foi rever sistematicamente a literatura, procurando identificar um corpo de evidências científicas que tenha considerado o efeito da fadiga muscular nas respostas da PA pós-exercício. Esta revisão poderá trazer repercussões práticas para a prescrição do exercício de força em pacientes hipertensos, uma vez que poderá evidenciar benefícios do exercício de força conduzido até a fadiga muscular concêntrica, apesar do risco inerente desta prática durante a sua realização.

\section{MÉTODO}

A revisão sistemática foi realizada com base em artigos publicados em periódicos nacionais e internacionais, que analisaram a resposta da PA após o exercício de força em indivíduos hipertensos. Foram consultadas as bases de dados SciELO (Scientific Electronic Library Online), LILACS (Literatura Latino-Americana e do Caribe em Ciências da Saúde) e PubMed/ MedLine (National Library of Medicine), procurando artigos publicados até 2010 .

Para a pesquisa foram utilizadas associações de termos em português e inglês, respectivamente, como: pressão arterial/blood pressure, hipertensão/hypertension, exercício com resistência/ resistance exercise, treino com pesos/ weight training, exercício/exercise e exercício de força/strength exercise. Ademais, na pesquisa realizada na base de dados Pubmed/ 
MedLine foi utilizada a ferramenta de limites de pesquisa, utilizando as seguintes opções de limites activadas: Humans, Clinical Trial, Randomized Controlled Trial, Classical Article, Clinical Trial, Phase I, Clinical Trial, Phase II, Clinical Trial, Phase III, Clinical Trial, Phase IV, Comparative Study, Controlled Clinical Trial, Journal Article, Multicenter Study, Overall, English.

Inicialmente, a seleção dos artigos foi efectuada por análise dos títulos, a fim de verificar a adequação dos temas ao propósito da revisão. Quando a decisão não pôde ser tomada a partir dos títulos, foi realizada a leitura do resumo e, permanecendo a dúvida, uma análise completa do estudo foi realizada. Os artigos foram obtidos por meio digital e, quando indisponíveis, foram adquiridos em bibliotecas e/ou pelo serviço de comutação bibliográfica. Foram incluídos nesta revisão somente estudos originais, excepto revisões sistemáticas e metanálises, que verificaram as respostas da PA após o exercício de força. Os estudos deveriam considerar exclusivamente exercícios de força, conducentes ou não à fadiga, realizados com hipertensos.

Os estudos que atenderam aos critérios de inclusão foram analisados de acordo com as seguintes variáveis: a) número de sujeitos no estudo; b) idade e gênero da amostra; c) estádio de hipertensão; d) nível de actividade física; e) medicação utilizada; f) número de séries ou circuitos; g) número de exercícios por grupo muscular; h) tempo de monitorização da PA; i) alterações na PA após o exercício; e j) se realizou ou não o exercício de força até a fadiga.

Todos os dados foram inseridos e quantificados numa folha de dados por pares. Além disso, considerou-se, por meio da escala PEDro (Maher, Sherrington, Herbert, Moseley, \& Elkins, 2003), específica para estudos clínicos, um índice qualitativo para a adequação dos estudos eleitos para a pesquisa. Essa escala consiste em 11 critérios que fornecem indicadores da validade interna dos estudos. Para atestar a concordância entre os avaliadores, procedeu-se a uma análise de concordância através do índice de Kappa e a respectiva classificação conforme sugerido por (Landis \& Koch, 1977), que considera $\mathrm{K} 0.00-0.19$ = pobre; $\mathrm{K}$ $0.20-0.39=$ regular, $\mathrm{K} 0.40-0.59=$ moderado; $\mathrm{K} 0.60$ - 0.79 = substancial; e $\mathrm{K} 0.80$ $1.00=$ quase perfeito. Para os artigos em que não houve consenso entre os dois avaliadores, um terceiro investigador fez a análise final.

\section{RESULTADOS}

Com a inserção dos descritores selecionados para a revisão, foram identificados 9377 estudos. Destes estudos, 8517 não estavam relacionados com atividade física e 575 não estavam relacionados com exercício de força. Cinquenta e nove estudos eram de revisão da literatura, 144 estudos não verificaram o efeito agudo do exercício na PA, e 69 estudos foram conduzidos com sujeitos normotensos. Devido a isso, conforme demonstrado na figura 1 , apenas seis estudos atenderam aos critérios estabelecidos e foram incluídos nesta revisão.

A tabela 1 apresenta informações sobre as características gerais dos seis estudos analisados. A análise da qualidade dos estudos demonstrou pontuações que variaram entre $5 \mathrm{a}$ 6 na escala PEDro, com concordância substancial entre os avaliadores $(\mathrm{K}=0.67 ; p=.08)$.

A maioria dos estudos foi publicada no ano de 2009 ( $n=3,50 \%)$ e envolveu indivíduos de ambos os gêneros $(n=4,66.7 \%)$. A média de idade dos indivíduos estudados variou de 43 a 66 anos. Apenas dois estudos (Hardy \& Tucker, 1998; Mota et al., 2009) relataram o estádio de hipertensão dos sujeitos. Quanto ao nível de atividade física, dois estudos (Mediano et al., 2005; Mota et al., 2009) incluíram indivíduos ativos fisicamente e dois estudos (Hardy \& Tucker, 1998; Marcos Doederlein Polito, Simão, Saccomani, \& Casonatto, 2009) incluíram indivíduos inativos fisicamente. No que concerne ao uso de medicação, dois estudos (Mota et al., 2009; Marcos Doederlein Polito et al., 2009) 


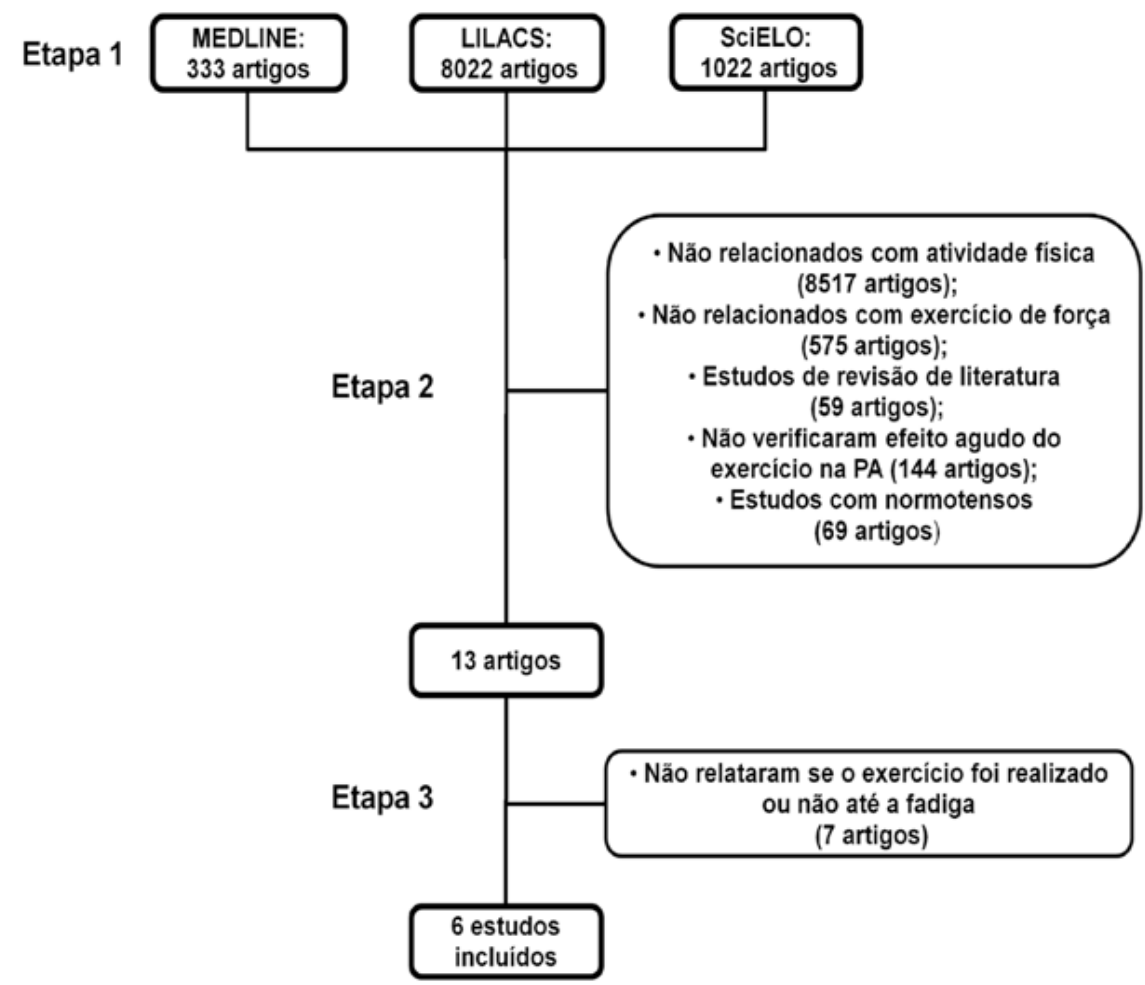

Figura 1: Fluxograma dos estudos incluídos na revisão

Tabela 1

Características dos indivíduos incluídos nos estudos de revisão

\begin{tabular}{ccccccc}
\hline Autor, ano & $n$ & $\begin{array}{c}\text { Idade } \\
\text { (média em anos) }\end{array}$ & Género & $\begin{array}{c}\text { Estádio de } \\
\text { hipertensão }\end{array}$ & $\begin{array}{c}\text { Nível de acti- } \\
\text { vidade física }\end{array}$ & Medicação \\
\hline Costa et al., 2010 & 15 & 66 & Feminino & NI & AF e IF & NI \\
Jannig et al., 2009 & 8 & 62 & Ambos & NI & NI & SC \\
Polito et al., 2009 & 12 & 51 & Ambos & NI & IF & $\begin{array}{c}\text { Betabloqueador, diuré- } \\
\text { tico, IECA } \\
\text { Beta-bloqueador, }\end{array}$ \\
Mota et al., 2009 & 15 & 43 & Ambos & Estágio I & AF & $\begin{array}{c}\text { Bloqueador de canal de } \\
\text { cálcio e IECA }\end{array}$ \\
Mediano et al., 2005 & 20 & 61 & Ambos & NI & AF & SC \\
Hardy e Tucker, 1998 & 24 & 51 & Masculino & Estágio I & IF & SM \\
\hline
\end{tabular}

NI - Não informado; AF - Ativos fisicamente; IF - Inativos fisicamente; SC - Sem controlo; IECA - Inibidor da enzima conversora da angiotensina; SM - Sem medicação.

incluíram indivíduos medicados com inibidores da enzima conversora da angiotensina e betabloqueador e dois estudos (Jannig, Cardoso, Fleischmann, Coelho, \& Carvalho, 2009; Mediano et al., 2005) não controlaram a medicação.

$\mathrm{Na}$ tabela 2 são apresentadas as informações sobre as características do exercício de força empregadas, bem como as respostas da PA sistólica e diastólica, verificadas pelos estudos.

Em todos os estudos, as sessões contem- plaram exercícios de força para membros superiores e inferiores. A maioria dos estudos (Costa, Gerage, Gonçalves, Pina, \& Polito, 2010; Jannig et al., 2009; Mediano et al., 2005) monitorizou a PA por um período de 60 minutos, sendo que cinco deles (Costa et al., 2010; Jannig et al., 2009; Mediano et al., 2005; Mota et al., 2009; Marcos Doederlein Polito et al., 2009) verificaram redução na PA sistólica ou diastólica.

Quatro estudos (Costa et al., 2010; Hardy 


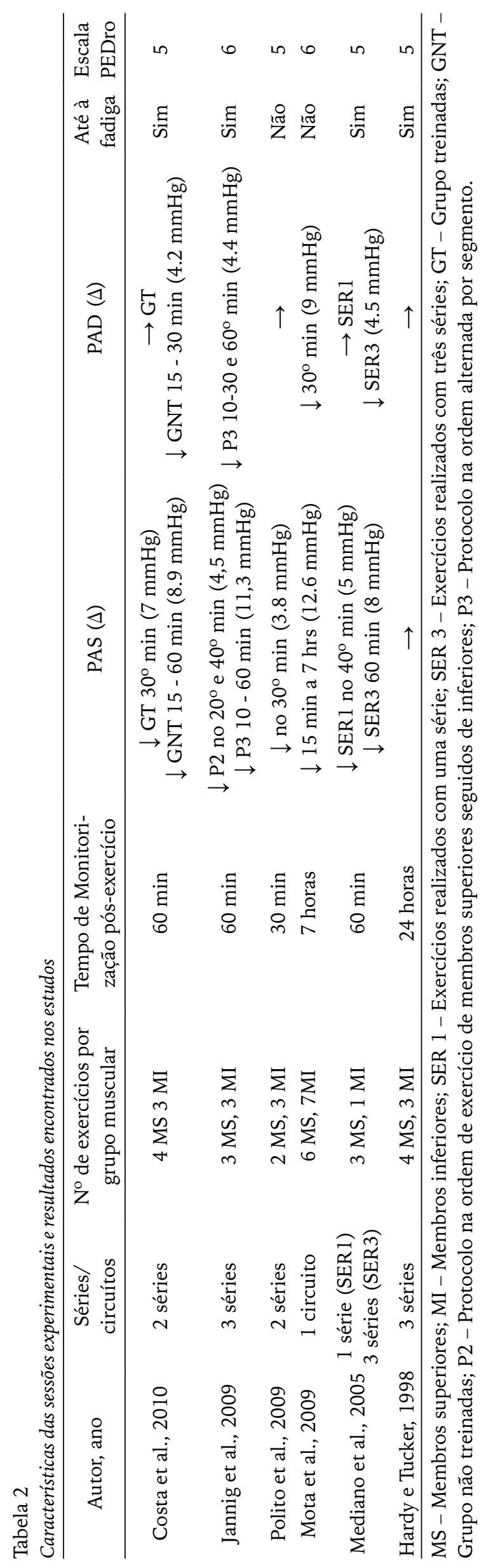

\& Tucker, 1998; Jannig et al., 2009; Mediano et al., 2005) relataram que o exercício de força foi realizado até a fadiga, ao passo que os demais (Mota et al., 2009; Marcos Doederlein Polito et al., 2009) relataram que os exercícios foram interrompidos antes da fadiga. Dos quatro estudos (Costa et al., 2010; Hardy \& Tucker, 1998; Jannig et al., 2009; Mediano et al., 2005) que utilizaram protocolos até a fadiga, três (Costa et al., 2010; Jannig et al., 2009; Mediano et al., 2005) observaram redução na PA com magnitude variando entre 4.5 a $11.3 \mathrm{mmHg}$. Por outro lado, quando o exercício foi interrompido antes da fadiga, todos relataram a ocorrência da redução na PA com magnitude variando entre 3.8 a $12.6 \mathrm{mmHg}$.

\section{DISCUSSÃO}

Os principais resultados desta revisão foram: (i) a maioria dos estudos realizou séries até a fadiga com sujeitos hipertensos; (ii) ambos os protocolos de exercício evidenciam redução da PA; e (iii) a magnitude da redução da PA não parece ser influenciada pela indução da fadiga.

Através da revisão sistemática foi possível observar que as características das amostras dos estudos foram amplamente diversificadas. Os estudos incluíram adultos e idosos, de ambos os gêneros, com diferentes níveis de atividade física e tipos de medicação utilizados. Todas estas variáveis devem ser consideradas na interpretação dos resultados, uma vez que estudos anteriores têm demonstrado que as características dos indivíduos estudados podem influenciar as respostas da PA após o exercício de força. Nesse sentido, têm sido verificadas reduções mais acentuadas da PAD em mulheres (Collier, 2008). No entanto, em nenhum estudo da presente revisão sistemática, houve a comparação da influência do gênero na resposta da PA pós-exercício de força. Além do mais, a magnitude da redução da PA parece ser maior com maiores valores de PA em repouso (Queiroz, Gagliardi, Forjaz, \& Rezk, 2009), de modo que se espera que o indivíduo 
com estadiamento de hipertensão mais acentuado apresente maior magnitude de redução da pressão arterial. Já a interveniência medicamentosa pode contribuir para a maior duração da redução da pressão arterial pós-exercício de força (Melo, Alencar Filho, Tinucci, Mion, \& Forjaz, 2006), porém pelo nosso conhecimento isto somente foi testado com o uso do captopril, de modo que a interveniência de outras classes medicamentosas ainda precisa ser devidamente investigada. Por fim, outro fator importante que pode influir nas respostas de PA pós-exercício é o tempo de engajamento na prática de exercícios, de forma que quanto mais treinado o indivíduo, menor será a alteração da PA pós-exercício (Costa et al., 2010). Assim, é possível que cada uma destas variáveis tenha influenciado as respostas da PA nos estudos da presente revisão, além da fadiga muscular.

Em relação à prescrição do exercício de força, todos os estudos realizaram exercícios para membros inferiores e superiores com séries múltiplas. Esta homogeneidade entre os estudos decorre, possivelmente, da utilização das recomendações do American College of Sports Medicine (1998) para a prescrição do exercício de força. Além disso, as evidências disponíveis na literatura até ao presente momento apontam para que a quantidade de massa muscular envolvida durante a realização do exercício, bem como o volume total realizado, interferiram nas respostas cardiovasculares pós-exercício, de forma que, quanto maior a massa muscular exercitada (Lizardo \& Simões, 2005) e número de séries (Mediano et al., 2005), maior redução na PA. Assim, pensa-se que os estudos incluídos nesta revisão procuraram utilizar protocolos consagrados previamente na literatura, aumentando assim a possibilidade de evidenciar diminuição da PA.

Após a pesquisa bibliográfica nas bases de dados nacionais e internacionais foram identificados apenas seis estudos que reportaram se o exercício de força foi prescrito até a fadiga, indicando que a maioria dos estudos sobre as respostas da PA após o exercício de força não controlou essa variável. Além do mais, não foi possível identificar nenhum estudo que almejou comparar as respostas cardiovasculares após o exercício de força até a fadiga e interrompido antes da fadiga, sendo identificados somente estudos que utilizaram apenas uma das formas de prescrição. Os resultados desta revisão demonstraram que tanto os estudos que realizaram o exercício de força até a fadiga, como os que interromperam o exercício antes da fadiga evidenciaram redução da PA. Estes resultados indicam que a ocorrência de fadiga no exercício de força parece não ser uma condição para a diminuição da PA. Na prática, estes resultados indicam que a interrupção do exercício antes da fadiga pode ser uma estratégia interessante em indivíduos com alto risco cardiovascular, uma vez que a redução da PA ocorre sem se verificarem aumentos exacerbados da PA durante o exercício de força.

Além disso, através desta revisão também foi possível observar que a magnitude da diminuição da PA observada nos dois protocolos de exercício foi similar. No entanto, estes resultados devem ser analisados com cautela, visto que tanto as características dos indivíduos quanto os protocolos utilizados foram diversificados entre os estudos. Perante esta evidência, é possível que a magnitude da redução da PA nos estudos tenha sido conseqüência de uma interação destas variáveis e não exclusivamente ocasionada pela realização ou não do exercício de força até a fadiga, tendo em vista a influência de cada uma delas nas respostas da PA, anteriormente relatadas.

Um aspecto interessante observado nesta revisão foi que nenhum dos estudos incluídos analisou as respostas da frequência cardíaca após o exercício de força. Trata-se de uma variável importante para hipertensos, pois está diretamente relacionada com a sobrecarga e com o risco cardiovasculares (Curtis \& O'Keefe, 2002). Aumentos da frequência cardíaca, mesmo que associados com a redução da PA 
podem ocasionar aumento do duplo produto, indicando assim o aumento da sobrecarga cardiovascular após o exercício. Desta forma, é possível que a realização do exercício até à fadiga influencie as respostas da frequência cardíaca após o exercício de força. Deste modo, estudos futuros são necessários para elucidar o impacto da fadiga na magnitude das respostas da PA após o treino de força, controlando variáveis importantes como frequência cardíaca, faixas etárias, tipos de medicações e diferentes níveis de actividade física.

Este estudo apresenta algumas limitações que precisam ser consideradas. A pesquisa bibliográfica restringiu-se a três bases de dados. Consequentemente, periódicos indexados em outras bases de dados não foram incluídos. Para a pesquisa bibliográfica foram utilizados apenas termos ou descritores em português ou inglês e, possivelmente, alguns estudos não foram incluídos por serem publicados em outros idiomas.

\section{CONCLUSÕES}

Os estudos evidenciaram que a redução da PA, bem como sua magnitude, após o treino de força em hipertensos é independente da realização do exercício até a fadiga, o que carece de ser analisado com cautela devido às diferenças metodológicas entre os estudos. Assim, sugere-se a realização de estudos futuros que analisem tanto o impacto da fadiga na magnitude das respostas da PA após o treino de força, como também em diferentes faixas etárias, medicações e níveis de treinamento dos sujeitos.

\section{Agradecimentos:}

Os autores agradecem a CAPES pela bolsa de mestrado outorgada.

\section{Conflito de Interesses:}

Nada declarado.
Nada declarado.

\section{REFERÊNCIAS}

Collier, S. R. (2008). Sex differences in the effects of aerobic and anaerobic exercise on blood pressure and arterial stiffness. Gend Med, 5(2), 115-123.

Costa, J. B. Y., Gerage, A. M., Gonçalves, C. G. S., Pina, F. L. C., \& Polito, M. D. (2010). Influência do Estado de Treinamento Sobre o Comportamento da Pressão Arterial Após uma Sessão de Exercícios com Pesos em Idosas Hipertensas. Revista Brasileira de Medicina do Esporte, 16(2), 103-106

Curtis, B. M., \& O’Keefe, J. H., Jr. (2002). Autonomic tone as a cardiovascular risk factor: the dangers of chronic fight or flight. Mayo Clinic Proceedings, $77(1), 45-54$.

de Souza Nery, S., Gomides, R. S., da Silva, G. V., de Moraes Forjaz, C. L., Mion, D., Jr., \& Tinucci, T. (2010). Intra-arterial blood pressure response in hypertensive subjects during low- and high-intensity resistance exercise. Clinics (Sao Paulo), 65(3), 271-277.

Gomides, R. S., Nery, S. d. S., Júnior, D. M., Tinucci, T., \& Forjaz, C. L. d. M. (2007). Pressão arterial durante o exercício resistido de diferentes intensidades em indivíduos hipertensos. Coleção Pesquisa em Educação Física, 6(1), 435-442.

Hardy, D. O., \& Tucker, L. A. (1998). The effects of a single bout of strength training on ambulatory blood pressure levels in 24 mildly hypertensive men. American Journal of Health Promotion, 13(2), 69-72.

Jannig, P. R., Cardoso, A. C., Fleischmann, E., Coelho, C. W., \& Carvalho, T. d. (2009). influência da ordem de Execução de Exercícios Resistidos na hipotensão Pós-exercício em idosos hipertensos. Revista Brasileira de Medicina do Esporte, 15(5), 338-341.

Landis, J. R., \& Koch, G. G. (1977). The measurement of observer agreement for categorical data. Biometrics, 33(1), 159-174.

Lizardo, J. H. d. F., \& Simões, H. G. (2005). Efeitos de diferentes sessões de exercícios resistidos 
sobre a hipotensão pós-exercício. Revista brasileira de fisioterapia, 9(3), 289-295.

Maher, C. G., Sherrington, C., Herbert, R. D., Moseley, A. M., \& Elkins, M. (2003). Reliability of the PEDro scale for rating quality of randomized controlled trials. Physical Therapy, 83(8), 713-721.

Maior, A. S., Jr., C. L. A., Ferraz, F. M., Menezes, M. d., Carvalheira, S., \& Simão, R. (2007). Efeito Hipotensivo dos Exercícios Resistidos Realizados em Diferentes Intervalos de Recuperação. Revista da SOCERJ, 20(1), 53-59.

Mediano, M. F. F., Paravidino, V., Simão, R., Pontes, F. L., \& Polito, M. D. (2005). Comportamento subagudo da pressão arterial após o treinamento de força em hipertensos controlados. Revista Brasileira de Medicina do Esporte, 11(6), 337-340.

Melo, C. M., Alencar Filho, A. C., Tinucci, T., Mion, D., Jr., \& Forjaz, C. L. (2006). Postexercise hypotension induced by low-intensity resistance exercise in hypertensive women receiving captopril. Blood Pressure Monitoring, 11(4), 183-189.

Mota, M. R., Pardono, E., Lima, L. C., Arsa, G., Bottaro, M., Campbell, C. S., et al. (2009). Effects of treadmill running and resistance exercises on lowering blood pressure during the daily work of hypertensive subjects. Journal of Strength and Conditioning Research, 23(8), 23312338.
Polito, M. D., \& Farinatti, P. T. (2009). The effects of muscle mass and number of sets during resistance exercise on postexercise hypotension. Journal of Strength and Conditioning Research, 23(8), 2351-2357.

Polito, M. D., Simão, R., Saccomani, M. G., \& Casonatto, J. (2009). Influência de uma Sessão de Exercício Aeróbio e Resistido sobre a Hipotensão Pós-Esforço em Hipertensos. Revista da SOCERJ, 22 (5), 330-334.

Queiroz, A. C., Gagliardi, J. F., Forjaz, C. L., \& Rezk, C. C. (2009). Clinic and ambulatory blood pressure responses after resistance exercise. Journal of Strength and Conditioning Research, 23(2), 571-578.

Rezk, C. C., Marrache, R. C., Tinucci, T., Mion, D., Jr., \& Forjaz, C. L. (2006). Post-resistance exercise hypotension, hemodynamics, and heart rate variability: influence of exercise intensity. European Journal of Applied Physiology, 98(1), 105-112. Veloso, J., Polito, M. D., Riera, T., Celes, R., Vidal, J. C., \& Bottaro, M. (2010). Effects of rest interval between exercise sets on blood pressure after resistance exercises. Arquivos Brasileiros de Cardiologia, 94(4), 512-518.

Willardson, J. M. (2007). The application of training to failure in periodized multiple-set resistance exercise programs. J Strength Cond Res, 21(2), 628-631.

(cc) EY-No Todo o conteúdo da revista Motricidade está licenciado sob a Creative Commons, exceto quando especificado em contrário e nos conteúdos retirados de outras fontes bibliográficas. 